Dieses Dokument ist eine Zweitveröffentlichung von:

Manuel Burghardt:

Digital Humanities in der Musikwissenschaft -

Computergestützte Erschließungsstrategien und Analyseansätze für handschriftliche Liedblätter

Veröffentlicht in:

BIBLIOTHEK - Forschung und Praxis, 42(2), S. 324-332.

Verlag: De Gruyter | Jahr: 2018

DOI: https://doi.org/10.1515/bfp-2018-0032

Es gelten die Regelungen des Urheberrechts, https://rightsstatements.org/page/InC/1.0/?language =de

Bereitgestellt von:

musiconn.publish - https://musiconn.qucosa.de 
Manuel Burghardt*

\section{Digital Humanities in der Musikwissenschaft - Computergestützte Erschließungsstrategien und Analyseansätze für handschriftliche Liedblätter}

https://doi.org/10.1515/bfp-2018-0032

Zusammenfassung: Der Beitrag beschreibt ein laufendes Projekt zur computergestützten Erschließung und Analyse einer großen Sammlung handschriftlicher Liedblätter mit Volksliedern aus dem deutschsprachigen Raum. Am Beispiel dieses praktischen Projekts werden Chancen und Herausforderungen diskutiert, die der Einsatz von Digital Humanities-Methoden für den Bereich der Musikwissenschaft mit sich bringt.

Schlüsselwörter: Digital Humanities; Crowdsourcing; Optical Music Recognition; Music Information Retrieval; Melodic Similarity

\section{Digital Humanities in Musicology - Toward Computer- based Transcription and Analysis of Handwritten Music Scores}

Abstract: This article presents an ongoing project for the computer-based transcription and analysis of handwritten music scores from a large collection of German folk tunes. Based on this project, I will discuss the challenges and opportunities that arise when using Digital Humanities methods in musicology.

Keywords: Digital Humanities; Crowdsourcing; Optical Music Recognition; Music Information Retrieval; Melodic Similarity

\section{Einleitung}

Die Verfügbarkeit und der Einsatz von digitaler Technologie in nahezu allen Bereichen des öffentlichen und privaten Lebens ändert auch in fundamentaler Weise wissenschaftliche Methoden und Forschungspraxen und läutet

*Kontaktperson: Manuel Burghardt, burghardt@informatik.uni-leipzig.de damit den Computational Turn ${ }^{1}$ ein. In zunehmendem Maße wird die wissenschaftliche Arbeit durch digitale Technologien vermittelt und beeinflusst, was sich nach und nach auch auf die epistemologischen und ontologischen Grundannahmen der einzelnen Disziplinen auswirkt. ${ }^{2}$ Mit der steigenden Verfügbarkeit digitalisierter Kulturartefakte in unterschiedlichsten Medienbereichen - sei es Text, Bild, Musik oder Video - und damit einhergehender computergestützter Analyseansätze haben sich im Spannungsfeld von Geisteswissenschaften und Informatik die Digital Humanities als eigener Teilbereich herausgebildet.

Während der Einsatz computergestützter Methoden in Fächern wie der Sprachwissenschaft (vgl. etwa die Korpuslinguistik) und der Literaturwissenschaft (vgl. Distant Reading $^{3}$ ) durch die Möglichkeit der unmittelbaren, maschinellen Verarbeitung des Untersuchungsgegenstands Text naheliegend ist, so sind in zunehmendem Maße auch Hinwendungen $\mathrm{zu}$ anderen, komplexeren Medientypen zu beobachten. Als Indiz hierfür mag etwa die neuerliche Gründung einer DHd-Arbeitsgruppe „Film und Video“4 sowie die Beantragung eines DFG-Schwerpunktprogramms „Das digitale Bild“5 gesehen werden. Für den Bereich der digitalen Musikwissenschaft reichen die Aktivitäten dabei durchaus weiter, mindestens bis in die Mitte der 1980erJahre, zurück. ${ }^{6}$ Aktuell ist die Musikwissenschaft in den Digital Humanities vor allem im Bereich der digitalen Edi-

1 Berry, David M.: The Computational Turn. Thinking About the Digital Humanities. In: Culture Machine 12 (2011).

2 Berry (Anm. 1).

3 Moretti, Franco: Conjectures on world literature. In: New Left Review 1 (2000), 54-68.

4 DHd ist der Name des offiziellen Fachverbands „Digital Humanities im deutschsprachigen Raum“. Informationen zur DHd-Arbeitsgruppe „Film und Video“ finden sich online unter http://dig-hum.de/ag-fil m-und-video. (Sämtliche URLs in diesem Artikel wurden zuletzt am 15.12.2017 überprüft.)

5 Vgl. http://www.kunstgeschichte.uni-muenchen.de/forschung/di gitalekg/digitales-bild1/index.html.

6 Vgl. die Schriftenreihe „Computing in Musicology“; Übersicht zu allen Ausgaben online verfügbar unter http://www.ccarh.org/publica tions/books/cm. 
tionen sehr stark vertreten. ${ }^{7}$ In diesem Beitrag sollen nun Möglichkeiten und Herausforderungen von Digital-Humanities-Verfahren für die Musikwissenschaft beleuchtet werden, die stärker in den Bereich der computergestützten Analyse von symbolischer Musik ${ }^{8}$ gehen und dabei Anleihen im benachbarten Feld des Music Information Retrieval (MIR) nehmen, das traditionell bei der Informatik sowie auch der Informationswissenschaft angesiedelt ist.

Eine wesentliche Voraussetzung für derartige digitale Analyseansätze ist die vorherige Erschließung und formale Repräsentation der Musikdaten, um diese einer maschinellen Verarbeitung überhaupt erst zugänglich zu machen. Am Fallbeispiel einer großen, handschriftlichen Sammlung volkstümlicher Liedblätter aus dem deutschsprachigen Raum (vgl. Abschnitt 2) sollen grundlegende Methoden zur Erschließung und Analyse von notierter Musik aufgezeigt werden. Zum Thema Erschließung werden im dritten Abschnitt Tools aus dem Bereich der Optical Music Recognition (OMR) diskutiert, die allerdings für handschriftlich notierte Musik nur begrenzt einsetzbar sind. Als alternative Erschließungsstrategie wird ein Crowdsourcing-Ansatz zur Transkription handschriftlicher Melodien mit dem selbst erstellten Tool Allegro vorgestellt. Im vierten Abschnitt schließlich werden Möglichkeiten einer computergestützten Analyse der zuvor digitalisierten Musikdaten aufgezeigt und dabei grundlegende Konzepte des MIR, insbesondere der Melodic Similarity, erörtert. Der Beitrag schließt mit einem Fazit zum Potenzial von DigitalHumanities-Ansätzen in der Musikwissenschaft.

\section{Fallstudie: Regensburger Liedblattsammlung}

Die Universitätsbibliothek Regensburg betreut seit 2001 Bestände (Text, Audio, Bild) aus mehreren Sammlungen zur Volksmusikforschung. ${ }^{9}$ Einen wesentlichen Teilbereich stellt eine umfangreiche Sammlung handschriftlicher Liedblätter aus dem deutschsprachigen Raum dar, deren Geschichte sich bis in das Jahr 1914 zurückverfolgen

7 Vgl. etwa das Projekt „Beethovens Werkstatt“, in dem mithilfe von genetischer Textkritik einerseits und digitaler Edition andererseits der komplexe kompositorische Prozess im Werk Beethovens untersucht werden soll. Online verfügbar unter http://beethovens-werkstatt.de. 8 Symbolisch notierte Musik stellt eine wichtige Abgrenzung zu Musik als akustischem Phänomen, das beispielsweise mit Methoden der Signalverarbeitung untersucht werden kann, dar.

9 Vgl. hierzu die Angaben des Regensburger Volksmusikportals (RVP), online unter http://rvp.ur.de/RVP.php. lässt. Damals wurde mit der Gründung des Deutschen Volksliedarchivs (DVA) in Freiburg im Breisgau erstmals der Versuch unternommen, eine systematische Sammlung deutscher Volkslieder zu etablieren. Die Regensburger Liedblattsammlung besteht aus ca. 140000 Blättern mit handschriftlich notierten Volksliedern aus dem gesamten deutschsprachigen Raum. Die Bestände gliedern sich in 18 Teilsammlungen, wobei die sogenannte A-Sammlung (handschriftlich überlieferte Volks- und Kinderlieder), mit etwa 120000 Blättern, die umfangreichste Teilsammlung ist. Neben den monophonen Melodien enthalten die Blätter zudem - meist mit Schreibmaschine verfasste - Liedtexte sowie einfache Metadaten, beispielsweise eine Liedblattnummer oder Angaben zum Archiv-Standort (vgl. Abb. 1). ${ }^{10}$ Ausführlichere Metadaten, wie beispielsweise Titel, Text-Incipit, Sangesort und Jahr, liegen in einem umfangreichen Zettelkastensystem vor; sie konnten im Rahmen von Vorarbeiten auf manuellem Wege bereits vollständig in eine elektronische Datenbank (Augias) übertragen werden.

\section{Maschinenlesbare Erschließung handschriftlicher Liedblätter}

Wenngleich im Rahmen des Regensburger Volksmusikportals schon jetzt viele der Liedblätter online anhand der bereits digitalisierten Metadaten in einem virtuellen Findbuch recherchierbar sind (vgl. Abb. 2), so ist das langfristige Ziel eine vollständig maschinenlesbare Repräsentation aller Melodien und Liedtexte. Mit einer derart erschlossenen Liedblattsammlung kann dann nicht nur ein komplexes Informationssystem zur Suche nach Text und Melodien umgesetzt werden, sondern es können auch computergestützte Analysen der Bestände im Sinne der Digital Humanities unternommen werden (vgl. Abschnitt 5). Nachfolgend werden einige Herausforderungen bei der Erschließung der handschriftlichen Melodien beschrieben.

10 Die hier wiedergegebenen Informationen zur Geschichte und Systematisierung der Liedblattsammlung stammen im Wesentlichen aus: Krüger, Gerd: Das „Regensburger Volksmusik-Portal“ der Universitätsbibliothek Regensburg. Bestände - Problematiken - Perspektiven. Zwischenbericht aus einem Erschließungsprojekt. In: Ruth-Elisabeth Mohrmann (Hrsg.): Audioarchive - Tondokumente digitalisieren, erschließen und auswerten, Münster u.a. 2013 (Beiträge zur Volkskultur in Nordwestdeutschland; 121) 119-31. 


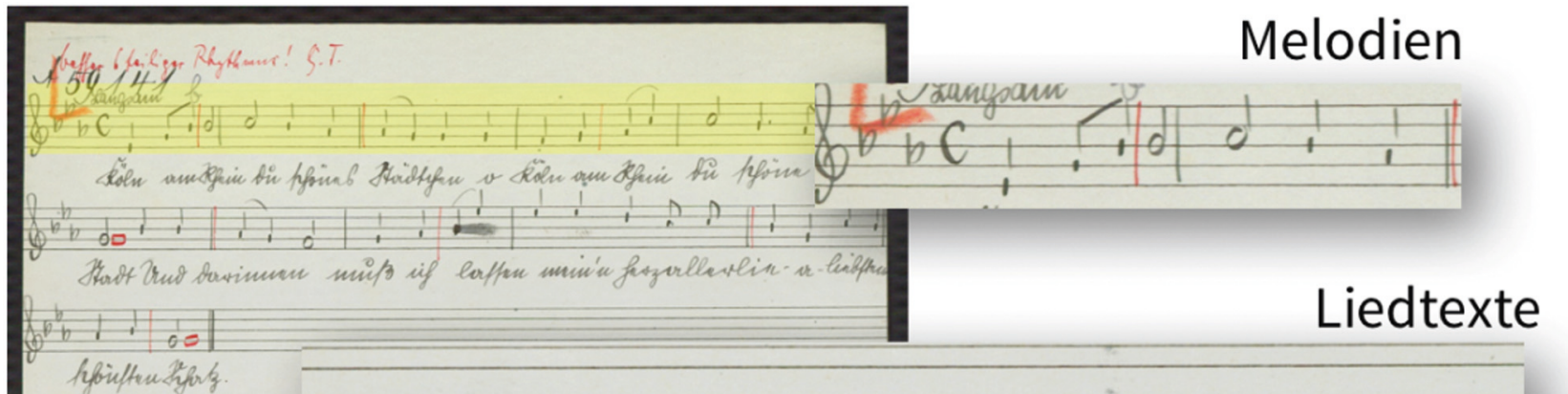

2. Sohatz, zoh Viel teusendme zonntest cu $\mathrm{m} 1$

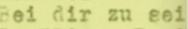
3. "D1eze Pre1 Bei mir zu sel. : Tienn an nir 318 ane die al 4. Pulver und Tonn man Franz I: Sehorne junge Genn man 10 5. An dem $1 \mathrm{~mm}$ Dio lounten holle :Der elne leveht' in mein enlaezirmer.

Der andre leucht' meim Suhatz naeh Raus:: !

Benern

2. Sehatz, ach Sehstz, du gust pish krthizen

Viel teusendmel in einer stund'.

Könntest du mir die Freiheit sehenken, Bei dir u eein, ja sein eine halbe stund': 3. "Diese Freiheit sollst du haben, Bei mir zu sein eine halbe stuna'. : Wenn du mir willst treu verbleiben 3 is auf die allerletzte stund!.: / Metadaten

Hessisches Archav A.17, II B1att 163

Abb. 1: Beispielhaftes Liedblatt aus der Regensburger Liedblattsammlung

\subsection{Optical Music Recognition für handschriftliche Liedblätter}

Aufgrund der großen Anzahl von Liedblättern bietet sich eine automatisierte Erschließung an. Analog zu bekannten Techniken aus der Texterkennung (Optical Character Recognition, OCR) finden sich auch für den Bereich der Notenerkennung Ansätze für Optical Music Recognition (OMR), ${ }^{11}$ bei denen versucht wird, durch Bilderkennung einzelne Noten automatisiert zu identifizieren. Wenngleich es eine ganze Reihe von verfügbaren OMR-Tools für die Erken- nung von gedruckten Noten gibt, so lassen die Ergebnisse hinsichtlich Präzision und Zuverlässigkeit häufig sehr zu wünschen übrig. ${ }^{12}$ Beim Versuch solch existierende OMRTools - die primär für die Erkennung gedruckter Noten konzipiert sind - auf handschriftliche Notenblätter anzuwenden, sind die Ergebnisse noch schlechter. Im Rahmen einer Evaluationsstudie ${ }^{14}$ für ein Testkorpus von 20 Liedblättern aus der Regensburger Sammlung wurden insgesamt drei verfügbare OMR-Tools evaluiert (vgl. Tab. 1).
11 Vgl. Bainbridge, David; Bell, Tim: The challenge of optical music recognition. In: Computers and the Humanities 35 (2001), 95-121. Homenda, Władysław; Luckner, Marcin: Automatic Knowledge Acquisition: Recognizing Music Notation with Methods of Centroids and Classifications Trees. In: The 2006 IEEE International Joint Conference on Neural Network Proceedings 48 (2006), 6414-20. Raphael, Christopher; Wang, Jingya: New Approaches to Optical Music Recognition. In: 12th International Society for Music Information Retrieval Conference (ISMIR) (2011), 305-10. Rebelo, Ana; Capela, Artur; Cardoso, Jaime S.: Optical recognition of music symbols. In: International Journal on Document Analysis and Recognition 13 (2010), 19-31.
12 Bellini, Pierfrancesco; Bruno, Ivan; Nesi, Paolo: Assessing Optical Music Recognition Tools Evaluation Methodologies for OMR Application. In: Computer Music Journal 31 (1) (2007), 68-93. Vgl. Artikel von Diet in diesem Heft S. 319-23.

13 Regensburger Volksmusikportal, Findbuch unter https://rvp.ur.d e/hoerburger/Augias/?Ob_Id=906607.

14 Burghardt, Manuel; Spanner, Sebastian; Schmidt, Thomas; Fuchs, Florian; Buchhop, Katia; Nickl, Miriam; Wolff, Christian: Digitale Erschließung einer Sammlung von Volksliedern aus dem deutschsprachigen Raum. In: Book of Abstracts, DHd 2017. 


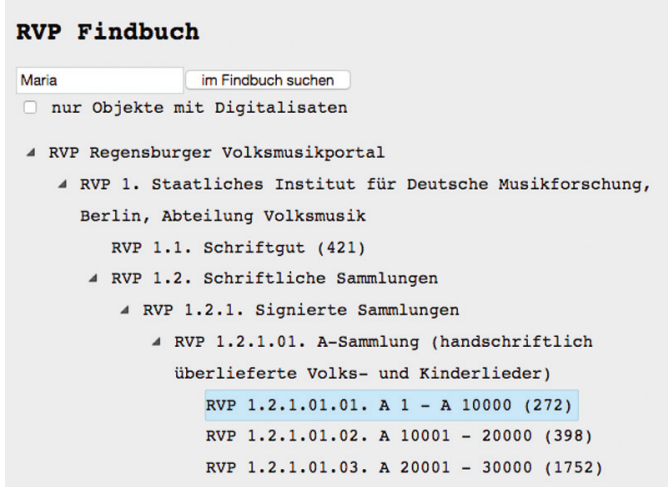

\begin{tabular}{|c|c|c|}
\hline 1. Num. & Titel & Dat. $\Rightarrow$ Findbuch \\
\hline A 1014 & Heimat ach Heimat, ich muss dich verlassen & ohne Datum \\
\hline A 1015 & Bei Sedan wohl auf der Höhe & ohne Datum \\
\hline A 1016 & Es gibt nicht Schönres auf der welt & ohne Datum \\
\hline A 1017 & Holdes grün wie lieb ich dich & ohne Datum \\
\hline A 1018 & Mein Regiment mein Heimatland & ohne Datum \\
\hline A 1019 & Wir sitzen so fröhlich beisammen & ohne Datum \\
\hline A 1020 & Hört ihr Jungfrauns Schreckenskunde, die sich zutrug in der Stadt & ohne Datum \\
\hline A 103 & Schönster Schatz du wirst erlauben, eine kleine viertelstund & ohne Datum \\
\hline A 1070 & Ich steh am eisernen Gegitter & 1900 \\
\hline
\end{tabular}

Abb. 2: Auszug aus dem Findbuch des Regensburger Volksmusikportals zur Recherche in der Liedblattsammlung ${ }^{13}$

Tab. 1: Überblick zu den evaluierten OMR-Tools

\begin{tabular}{ll}
\hline Name & Quelle \\
\hline CapellaScan & http://www.capella.de/de/index.cfm/produkte/ \\
& capella-scan/ \\
\hline PhotoScore & http://www.sibelius.com/products/photoscore/ulti- \\
& mate.html \\
\hline SharpEye & https://www.columbussoft.de/SharpEye.php \\
\hline
\end{tabular}

Das Evaluationsdesign und die verwendeten Metriken orientierten sich dabei an bestehenden Evaluationsstudien für OMR-Tools für gedruckte Noten. ${ }^{15}$ Die Ergebnisse der Evaluation zeigten, dass die durchschnittliche Erkennungsrate der getesteten Tools sehr gering (CapellaScan $8 \%$, PhotoScore $36 \%$, SharpEye $4 \%$ ) und damit nicht geeignet für den produktiven Einsatz für die Digitalisierung der Regensburger Liedblattsammlung ist.

\subsection{Crowdsourcing-Ansatz: Allegro}

Als alternativer Ansatz für die Erschließung der umfangreichen Liedblattsammlung wurde ein CrowdsourcingTool namens Allegro ${ }^{16}$ entwickelt. In der Literatur finden sich zahlreiche Belege für den erfolgreichen Einsatz von Crowdsourcing-Strategien im Bereich der Digital Humanities, ${ }^{17}$ insbesondere für Aufgaben im Bereich der Transkrip-

15 Bellini et al. (Anm. 13).

16 Allegro wurde von Sebastian Spanner im Rahmen seiner Masterarbeit entwickelt. Die Beta-Version ist verfügbar unter http:// 138.68.106.29.

17 Vgl. etwa Dunn, Stuart; Hedges, Mark: Connected Communities. Crowd-Sourcing in the Humanities. A Scoping Study. Connected Communities, 2012. Holley, Rose: Crowdsourcing: How and why should libraries do it? In: D-Lib Magazine 16 (3-4) (2010), http://doi.or g/10.1045/march2010-holley. tion, ${ }^{18}$ sowohl für die Transkription von Manuskripten ${ }^{19}$ als auch für den Anwendungsfall der Transkription von Notenblättern..$^{20} \mathrm{Zu}$ Beginn des Entwicklungsprozesses von Allegro wurde zunächst eine grundlegende Analyse vorhandener Transkriptionstools (vgl. Tab. 2) vorgenommen, einerseits um einen Überblick zu typischen Funktionen solcher Werkzeuge zu erhalten, andererseits um auszuloten, inwiefern bestehende Tools für den Einsatz in einem kollaborativen Crowdsourcing-Szenario geeignet sind.

Tab. 2: Überblick zu einigen bekannten Tools für die Notation und Transkription von Musikdaten

\begin{tabular}{lll}
\hline Name & Typ & Quelle \\
\hline Flat.io & Web & https://flat.io/ \\
\hline Noteflight & Web & https://www.noteflight.com \\
\hline Sibelius & Desktop & http://www.avid.com/en/sibelius \\
\hline MuseScore & Desktop & https://musescore.org/ \\
\hline
\end{tabular}

Da keines der existierenden Tools unmittelbar für Crowdsourcing ausgelegt ist, wurde mit Allegro ein neues Konzept entwickelt, das explizit bestehende best practices aus erfolgreichen Crowdsourcing-Projekten umsetzt. ${ }^{21}$ Alle-

18 Oomen, Johan; Aroyo, Lora: Crowdsourcing in the Cultural Heritage Domain. Opportunities and Challenges. In: $C \& T$ '11 Proceedings of the 5th International Conference on Communities and Technologies (July) (2011), 138-49.

19 Causer, Tim; Wallace, Valerie: Building A Volunteer Community: Results and Findings from Transcribe Bentham. In: DHQ: Digital Humanities Quarterly 6 (2) (2012).

20 What's the Score? Online verfügbar unter https://www.bodleian. ox.ac.uk/weston/our-work/projects/whats-the-score.

21 Burghardt, Manuel; Spanner, Sebastian: Allegro: User-centered Design of a Tool for the Crowdsourced Transcription of Handwritten Music Scores. In: Proceedings of the DATeCH (Digital Access to Textual Cultural Heritage) conference. ACM (2017). 


\section{1) Taktsegmentierung}

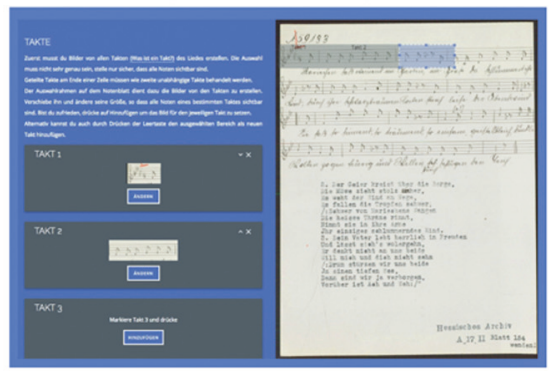

2) Metadaten

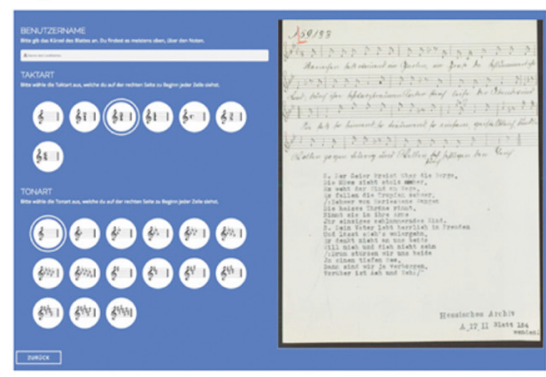

3) Transkription

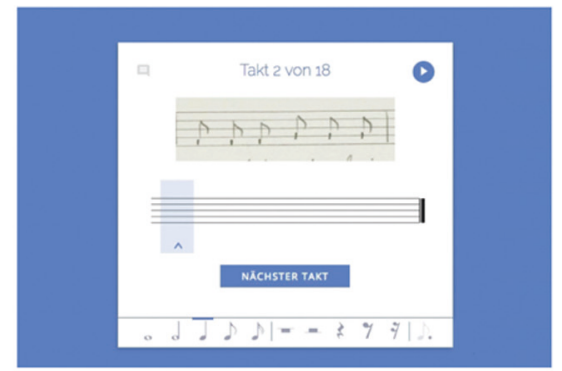

Abb. 3: Wesentliche Schritte bei der Transkription mit Allegro

gro wurde ausschließlich mit aktuellen Webtechnologien ${ }^{22}$ umgesetzt und kann ohne vorherige Installation direkt im Browser ausgeführt werden. Aufgrund der enormen Menge an Liedblättern wurde beim Design von Allegro insbesondere auch der Aspekt der Usability berücksichtigt. ${ }^{23}$ Dabei soll die Einstiegshürde für potenzielle Nutzer so gering wie möglich sein und die Bedienung des Tools so einfach gehalten werden, dass selbst Nutzer, die nicht aktiv Noten lesen können, in der Lage sind, die Blätter zu transkribieren. ${ }^{24}$ Zur Erreichung dieser Ziele kamen während des gesamten Entwicklungsprozesses systematisch Techniken des Usability Engineering und Ansätze aus dem Bereich des User Centered Design (UCD) zum Einsatz. ${ }^{25}$

Die Transkription mit Allegro gliedert sich im Wesentlichen in drei Phasen ${ }^{26}$ (vgl. Abb. 3): (1) Zunächst wird das Liedblatt in einzelne Takte segmentiert. Dieser Schritt ist wichtig, um später eine taktweise Transkription umsetzen zu können. Bereits in frühen Nutzerstudien mit dem Allegro-Prototyp wurde deutlich, dass eine Transkription Takt für Takt das Fehlerpotenzial gegenüber der Transkription des gesamten Liedblatts erheblich reduziert, da die Nutzer hier häufig den Überblick bzw. die Orientierung im Notenblatt verloren. (2) Im nächsten Schritt transkribieren die

22 Zum Einsatz kam ein sog. MEAN-Stack: Mongo DB als Datenbank, Express als Backend-Web-Framework, Angular.js als Frontend-Framework und Node.js als Backend-Laufzeitumgebung, vgl. http://mean. io.

23 Burghardt; Spanner (Anm. 21).

24 Meier, Florian; Bazo, Alexander; Burghardt, Manuel; Wolff, Christian: A Crowdsourced Encoding Approach for Handwritten Sheet Music. In: Roland, Perry; Kepper, Johannes (Hrsg.): Music Encoding Conference Proceedings 2013 and 2014 (2015), 127-30.

25 Vgl. etwa Nielsen, Jakob: Usability Engineering. Amsterdam u. a. 1993. Johnson, Jeff: Designing with the Mind in Mind. Simple Guide to Understanding User Interface Design Rules. Children. Amsterdam u. a. 2010. ISO 9241-210. Ergonomics of human-system interaction Part 210: Human-centred design process for interactive systems, 2010. 26 Ein kurzes Demovideo mit allen Phasen des Transkriptionsprozesses in Allegro findet sich online unter https://www.youtube.com/ watch?v=NllpocVRj48.
Anwender die Liedblatt-ID, die für eine spätere Verknüpfung mit den bestehenden Metadaten notwendig ist. Außerdem werden in diesem Schritt die Taktart und die Tonart angegeben. (3) Im letzten Schritt erfolgt die eigentliche Transkription der einzelnen Takte. Über ein OnboardingTutorial werden die Transkriptoren bei der ersten Nutzung auf die grundlegenden Funktionen hingewiesen. Die Transkription erfolgt durch Maus oder Tastatur in einer interaktiven Notenzeile. Das gesamte Lied kann nach der Transkription zu Testzwecken auch im Browser abgespielt werden, um so ggf. fehlerhafte Transkriptionen akustisch wahrnehmbar zu machen. ${ }^{27}$ Neben diesem einfachen Mechanismus zur grundlegenden Kontrolle der Transkriptionen durch die einzelnen Nutzer wird in Allegro darüber hinaus eine systematische Qualitätskontrolle im Sinne eines Double-Keying-Verfahrens ${ }^{28}$ durchgeführt. Dabei wird jedes Liedblatt von zwei unabhängigen Transkriptoren abgeschrieben. Falls es zu Abweichungen bei den beiden Transkriptionsvarianten kommt, wird das betreffende Liedblatt von einem Experten nochmals manuell geprüft. Allegro bietet hierfür eine spezielle Vergleichs- bzw. Korrekturansicht. Wenn die Transkriptionen zweier unabhängiger Nutzer vollständig übereinstimmen fällt der Schritt der manuellen Korrektur weg.

Durch den Web-Kontext des Tools werden zunächst alle Transkriptionen in MusicJSON, also einem Format basierend auf der JavaScript Object Notation, ${ }^{29}$ repräsentiert. Dieses Format kann mit entsprechenden Konvertierungstools aber problemlos in andere Music Encoding-Formate

$27 \mathrm{Zu}$ diesem Zwecke werden die Transkriptionen mit der JavaScriptBibliothek Midi.js in abspielbare Midi-Dateien umgewandelt. Midi.js ist online verfügbar unter https://galactic.ink/midi-js.

28 Haaf, Susanne; Wiegand, Frank; Geyken, Alexander: Measuring the correctness of double-keying. Error classification and quality control in a large corpus of TEI-annotated historical text. In: Journal of the Text Encoding Initiative (4) (2013). 29 JSON: https://www.json.org. 
wie beispielsweise MusicXML ${ }^{30}$ oder MEI (Music Encoding Initiative) $)^{31}$ übertragen werden.

Als Zwischenfazit zur Erschließung der Regensburger Liedblattsammlung kann festgehalten werden, dass sich ein toolgestützter Crowdsourcing-Ansatz grundsätzlich gut eignet, derartiges Material zu transkribieren. Dabei muss allerdings kritisch angemerkt werden, dass der Zeitfaktor für die korrekte Transkription aller Liedblätter der umfangreichen Sammlung trotz Crowdsourcing immer noch sehr hoch ist. Aktuell beträgt die durchschnittliche Transkriptionszeit pro Blatt ca. sechs Minuten. Bei zwei jeweils unabhängigen Transkriptionen für insgesamt 140000 Blätter kommt man somit auf 28000 Arbeitsstunden - die Korrektur der fehlerhaften Blätter nicht miteingerechnet. Somit ist das Ziel des Ansatzes vielmehr, mithilfe von Crowdsourcing ein ausreichend großen Trainingskorpus zu erstellen, um dann im nächsten Schritt mithilfe von maschinellem Lernen die restlichen Blätter automatisiert transkribieren zu lassen. $^{32}$

\section{Computergestützte Analyse von Liedblättern}

Neben vollständigen Musik-Editionen gehört der Vergleich einzelner Werke hinsichtlich melodischer Auffälligkeiten zu den typischen Forschungstätigkeiten der Musikwissenschaft. Dabei sind elektronische Datenbanken (vgl. Tab. 3), die eine Suche nach grundlegenden Metadaten und beispielsweise dem Incipit eines Stücks ermöglichen, bereits ein wichtiges Hilfsmittel, welches derartige Vergleiche deutlich erleichtert.

30 Good, Michael D.: MusicXML for Notation and Analysis. In: Hewlett, Walter B.; Selfridge-Field Eleanor (Hrsg.): The Virtual Score. Representation, Retrieval, Restoration, Stanford 2001 (Computing in Musicology; 12), 113-24.

31 Roland, Perry: The Music Encoding Initiative (MEI). In: Proceedings of the 1st International Conference on Musical Applications Using $X M L$ (2002), 50-55.

32 Für den Bereich der automatischen Handschriftenerkennung von Text (Handwritten Text Recognition, HTR) gibt es vielversprechende Ansätze, die in eine vergleichbare Richtung gehen. Vgl. hierzu etwa das Projekt Transkribus unter https://transkribus.eu/Transkribus und den Artikel von Rebulla et al. in diesem Heft S. 255-62.
Tab. 3: Music-Information-Retrieval-Systeme für Musikwissenschaftler (Auswahl)

\begin{tabular}{ll}
\hline Name & Quelle \\
\hline $\begin{array}{l}\text { Digital Archive of Finnish } \\
\text { Folk Song Tunes }\end{array}$ & http://esavelmat.jyu.fi/index_en.html \\
\hline Dutch Song Database & http://www.liederenbank.nl/ \\
\hline HymnQuest & http://hymnquest.com/ \\
\hline Humdrum & http://www.musiccog.ohio-state.edu/ \\
& Humdrum/ \\
\hline Melody Catcher & http://melodycatcher.com/ \\
\hline Musipedia & http://www.musipedia.org/ \\
\hline PeachNote & http://www.peachnote.com/ \\
\hline Probado & http://www.probado.de/ \\
\hline RISM & https://opac.rism.info/ \\
\hline Themefinder & http://www.themefinder.org/
\end{tabular}

Auch für die Regensburger Liedblattsammlung soll langfristig ein Informationssystem entstehen, das die Suche nach Melodiesequenzen in beliebigen Teilen des Stücks möglich macht. Darüber hinaus soll die Melodiesuche mit einer gewissen Unschärfe bzw. Abstraktion möglich sein, d.h., es sollen nicht nur exakte Übereinstimmungen bezüglich eines bestimmten Melodiefragments gefunden werden, sondern auch solche Lieder als Ergebnis zurückgegeben werden, die bis zu einem gewissen Grad ähnlich sind. Auf diese Weise können weiterreichende Zusammenhänge und Verwandtschaftsbeziehungen zwischen Melodien auf struktureller Ebene identifiziert werden. Diese Unschärfe bei der Suche nach Melodiefragmenten kann zum einen durch Abstraktion auf der Repräsentationsebene einer Melodie erfolgen. Zum anderen lässt sich mithilfe sogenannter Melodic Similarity-Algorithmen ${ }^{33}$ der Grad der Ähnlichkeit zweier Melodien graduell bestimmen.

33 Vgl. etwa Cahill, Margaret; Maidín, Donncha Ó: Melodic similarity algorithms - Using similarity ratings for development and early evaluation. In: Proceedings of the 6th International Conference on Music Information Retrieval (ISMIR) (2005), 450-53. Grachten, Maarten; Arcos, Josep Lluis; de Mántaras, Ramon Lopez: A comparison of different approaches to melodic similarity. In: Proceedings of the 2nd International Conference in Music and Artificial Intelligence (ICMAI) (2002). Miura, Takao; Shioya, Isamu: Similarity among melodies for music information retrieval. In: Proceedings of the 12th International Conference on Information and Knowledge Management (CIKM) (2003) 61-68. Müllensiefen, Daniel; Frieler, Klaus: Optimizing Measures of Melodic Similarity for The Exploration of a Large Folk Song Database. In: Proceedings of the 5th International Conference on Music Information Retrieval (ISMIR) (2004), 274-80. Müllensiefen, Daniel; Frieler, Klaus: Melodic Similarity. Approaches and Applications. In: Proceedings of the 8th International Conference on Music Perception \& Cognition (2004), 283-89. 
(1) Exakte Melodie

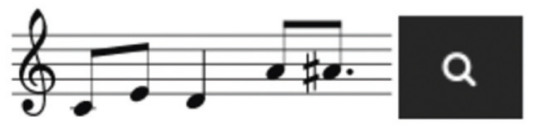

(2) Intervalle

$\begin{array}{llll}4 & 8 & 2 & -7\end{array}$

\section{(3) Parsons-Code}

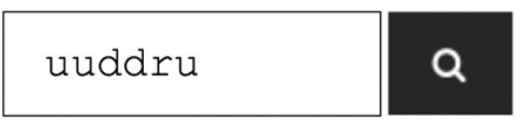

Abb. 4: Beispiele für unterschiedliche abstrakte Repräsentationen von Melodiesequenzen

\subsection{Abstraktion auf der Repräsentationsebene}

Üblicherweise werden in der Musikwissenschaft konkrete Melodiesequenzen, basierend auf der genauen Tonhöhe und -länge, miteinander verglichen. Versucht man melodische Ähnlichkeit hingegen aus psychoakustischer Perspektive zu operationalisieren, dann zeigen schon Wahrnehmungsexperimente aus den 1970er-Jahren, dass eine nicht hundertprozentige exakte Nachahmung einer Melodie von Probanden häufig nicht vom Original zu unterscheiden ist, wenn diese eine ähnliche Melodiekontur aufweisen. ${ }^{34}$ Eine Melodiekontur ist eine Abstraktion der eigentlichen Melodie, bei welcher der Faktor Tonlänge vollkommen ausgeblendet wird. Damit ist eine Melodiekontur eine bloße Sequenz von Intervallen oder Tonhöhen. Eine zusätzliche Abstraktion der ohnehin schon reduzierten Melodiekontur stellt der sogenannte Parsons-Code dar, bei dem schließlich auch die exakte Tonhöhe einzelner Noten ausgeblendet wird und stattdessen nur die Beziehung zweier aufeinanderfolgender Noten als Ansteigen ( $\mathrm{u}$ $=u p)$, Abfallen $(\mathrm{d}=$ down $)$ oder Gleichbleiben $(\mathrm{r}=$ repeat $)$ repräsentiert wird. Abb. 4 zeigt die beispielhafte Umsetzung von drei Suchanfragen (1) als exakte Melodie und als abstrakte Melodiekontur, (2) einmal als Abfolge von Intervallen (Anstieg/Abfallen der Tonhöhe durch Angabe von positiven/negativen Halbtonschritten) und einmal als (3) Parsons-Code, der nur das tendenzielle Ansteigen oder Abfallen einer Melodie berücksichtigt.

\subsection{Melodic-Similarity-Funktion}

Durch die Abstraktion der Repräsentationsebene können bereits weitreichende strukturelle Verwandtschaftsbeziehungen zwischen einzelnen Liedblättern identifiziert werden. Zusätzlich wird durch die Implementierung einer $\mathrm{Me}$ lodic-Similarity-Funktion ein Mechanismus zur graduellen

34 Dowling, W. Jay: Scale and contour: Two components of a theory of memory for melodies. In: Psychological Review 85 (4) (1978), 34154.
Erfassung melodischer Ähnlichkeit umgesetzt, der es beispielsweise erlaubt, Melodienfragmente, die mindestens eine Übereinstimmung von $75 \%$ aufweisen, zu identifizieren.

Ein Standard-Algorithmus zur Berechnung von melodischer Ähnlichkeit zwischen zwei Melodien ist der Mongeau-Sankoff-Algorithmus, ${ }^{35}$ der im Wesentlichen auf dem Prinzip der Editierdistanz basiert. Dabei errechnet sich die Distanz zwischen zwei Melodien aus der Anzahl der Editier-Operationen, ${ }^{36}$ die nötig sind, um eine Melodie in eine andere zu überführen. Zusätzlich führen Mongeau und Sankhoff zwei weitere Operationen „Fragmentierung“ (fragmentation) und „Konsolidierung“ (consolidation) ein. Die Fragmentierungsoperation berücksichtigt, dass eine Note aus der Quellsequenz durch mehrere Noten in der Zielsequenz ersetzt werden kann (beispielsweise eine ganze Note durch vier Viertelnoten), Konsolidierung beschreibt den umgekehrten Prozess der Ersetzung mehrerer Noten durch eine einzige Note. Die Details des Algorithmus sehen noch eine unterschiedliche Gewichtung der unterschiedlichen Operationen vor. Das Grundprinzip lässt sich folgendermaßen zusammenfassen: Je mehr Editieroperationen nötig sind, um eine Melodie in eine andere zu überführen, desto geringer ist deren Melodic Similarity Score.

\subsection{MIR-System für die Analyse der Liedblattsammlung}

Die beiden eben skizzierten Möglichkeiten der graduellen Identifikation melodischer Ähnlichkeit in Melodien wurden in einem funktionsfähigen Prototyp umgesetzt, welcher die Analyse eines kleinen Subkorpus an fertig transkribierten Liedblättern erlaubt. ${ }^{37}$ Mit dem System können

35 Mongeau, Marcel; Sankoff, David: Comparison of Musical Sequences. In: Computers and the Humanities 24 (1990), 161-75.

36 Die drei grundlegenden Operationen sind dabei: (1) neue Note einfügen, (2) bestehende Note löschen und (3) bestehende Note durch andere Note ersetzen.

37 Der Prototyp wurde von Lukas Lamm im Rahmen seiner Masterarbeit entwickelt. Demovideo unter https://vimeo.com/187879605. 


\section{Suche Parsons Code: uduuddudd}
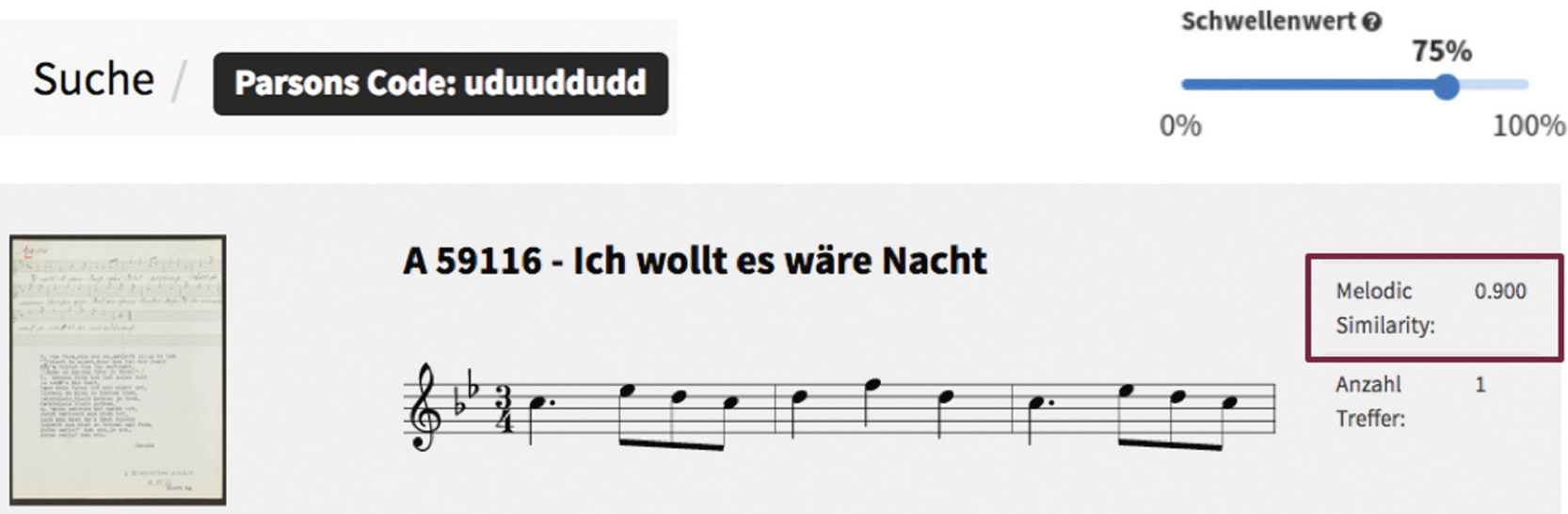

\section{A 59116 - Ich wollt es wäre Nacht}

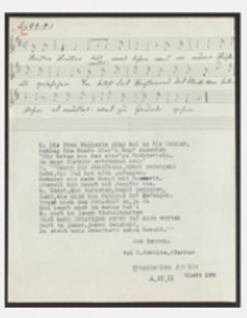

\section{A 59221 - Meister Müller tut mal sehen}
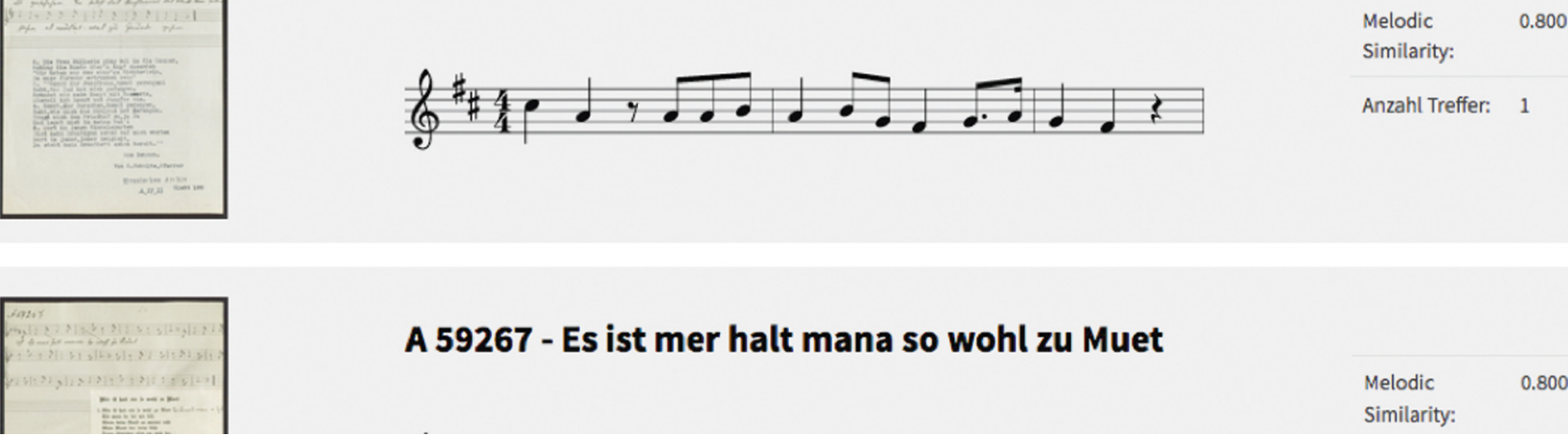

\section{A 59267 - Es ist mer halt mana so wohl zu Muet}

Abb. 5: Beispielhafte Suchanfrage im Parsons-Code und die resultierende Ergebnisliste

melodische Suchanfragen entweder als exakte Melodie, als Intervallfolge oder als Parsons-Code-Sequenz beantwortet werden. Abb. 5 zeigt ein beispielhaftes Szenario, in dem nach dem Parsons-Code ,up-down-up-up-down-down-updown-down" gesucht wird. In der Ergebnisliste sollen alle Liedblätter vorkommen, die diese Sequenz an irgendeiner Stelle der Melodie zu mindestens $75 \%$ enthalten. ${ }^{38}$

Jeder Treffer der Ergebnisliste kann näher untersucht werden. Dabei wird zunächst der Originalscan des Liedblatts angezeigt (vgl. Abb. 6). Darüber hinaus wird ein virtuelles Notenblatt anhand der Transkription dargestellt, in welchem die gesuchte Melodiesequenz entsprechend hervorgehoben ist. Sowohl die Transkription als auch ein synthetisiertes MIDI-File können heruntergeladen werden.

38 Eine Evaluation der implementierten Melodic-Similarity-Komponente findet sich in Burghardt, Manuel; Lamm, Lukas: Entwicklung eines Music Information Retrieval-Tools zur Melodic Similarity-Analyse deutschsprachiger Volkslieder. In: Eibl, Maximilian; Gaedke, Martin (Hrsg.): INFORMATIK 2017 (Lecture Notes in Informatics (LNI), Gesellschaft für Informatik - Workshop „Musik trifft Informatik“) Bonn 2017, 15-27.
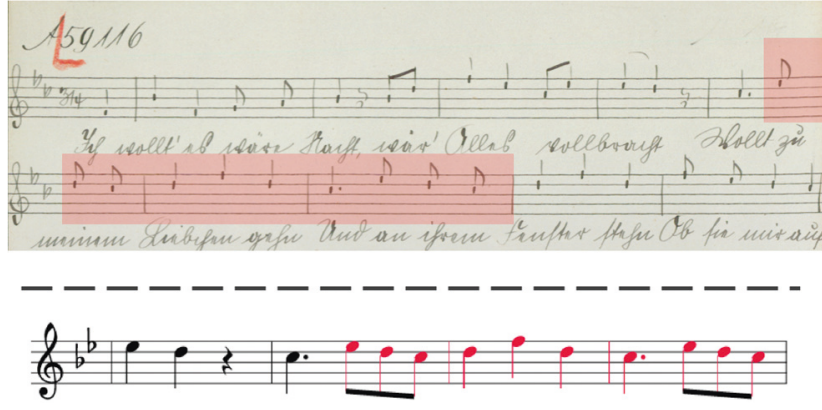

Abb. 6: Detailansicht eines Suchergebnisses

Als weitere Möglichkeit der Exploration der Liedblattsammlung anhand melodischer Ähnlichkeitsbeziehungen ist eine Netzwerkansicht gegeben. Dabei kann eine Auswahl von Liedblättern (beispielsweise aus einer bestimmten Region) als Netzwerk visualisiert werden, in dem jeder Knoten ein Liedblatt darstellt (vgl. Abb. 7). Klickt man ein Liedblatt in diesem Netzwerk an, so werden alle Liedblätter angezeigt, die eine melodische Ähnlichkeit zum Ausgangsliedblatt aufweisen, die größer als der definierte Schwellwert ist. 


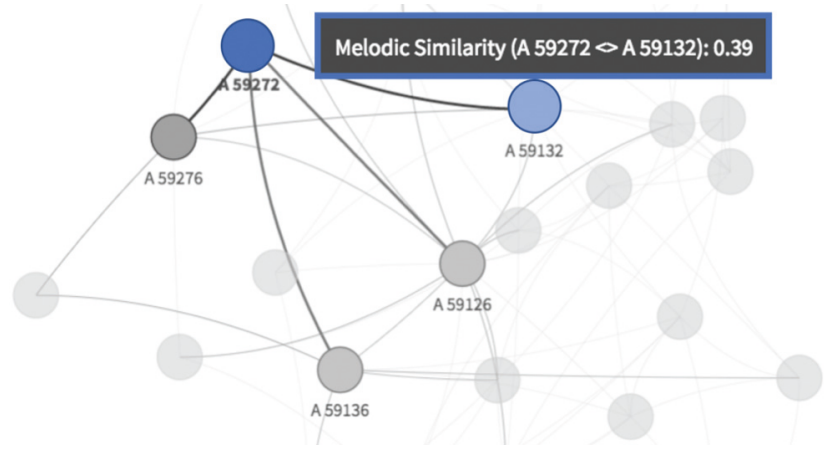

Abb. 7: Netzwerkdarstellung melodischer Ähnlichkeitsbeziehungen für ein exemplarisches Liedblattkorpus

\section{Fazit: Digital Humanities in der Musikwissenschaft}

Das prototypische Informationssystem, das im vorhergehenden Abschnitt vorgestellt wurde, kann als Machbarkeitsstudie verstanden werden, die illustriert, wie in großen digitalen Sammlungen computergestützt melodische Ähnlichkeiten identifiziert werden können. Dadurch ergeben sich aus musikwissenschaftlicher Perspektive interessante Möglichkeiten, das Phänomen Volkslied bzw. volkstümliche Musik empirisch an einem riesigen Korpus, ohne subjektiv-wertende Vorurteile zu untersuchen. Mithilfe des skizzierten Informationssystems können beispielsweise hochfrequente Muster in den Melodien automatisiert erkannt werden und im Sinne lokal begrenzter Topoi näher analysiert werden, etwa durch den Vergleich mit den vorhandenen Metadaten (Ort, Zeit). Gibt es beispielsweise auffällige Muster oder melodische Universalien für eine bestimmte Region oder ändern sich Melodiemuster im Laufe der Zeit, was deren Häufigkeitsverteilung angeht (diachrone Perspektive)? In Kombination mit den Liedtexten, die über vorhandene OCR-Tools gut erschlossen werden können, ergeben sich zudem spannende Möglichkeiten im Bereich melodisch-textueller Kollokationen: Kommen also bestimmte Schlüsselwörter (beispielsweise aus den semantischen Feldern Liebe, Tod, Geburt) besonders häufig mit bestimmten melodischen Mustern vor? In weiterführender Perspektive wäre auch denkbar, besonders hochfrequente Melodiesequenzen mit anderen Beständen abzugleichen, um beispielsweise herauszufinden, inwiefern sich die heutige volkstümliche Musik von den Volksliedern aus der Regensburger Liedblattsammlung abhebt.

Zum aktuellen Zeitpunkt mag das vorgestellte Projekt als Experiment gelten, in dem grundlegend untersucht wird, zu welchen (neuen) Erkenntnissen man mit quantita- tiven Ansätzen in der Musikwissenschaft überhaupt kommen kann. Der Bereich der volkstümlichen Musik scheint dabei besonders gut geeignet, da die relativ einfachen, monophonen Melodien wesentlich leichter als komplexere Werke der Kunstmusik formalisiert und quantitativ ausgewertet werden können. Der relativen Einfachheit der Melodien steht die enorme Quantität der einzelnen Liedblätter gegenüber, die mit qualitativen Methoden weder subjektiv noch intuitiv bewältigt werden kann. ${ }^{39}$ Damit sind Volkslieder die ideale Petrischale, um das Potenzial von DigitalHumanities-Ansätzen in der Musikwissenschaft grundlegend zu erproben. Berücksichtigt man zudem die zunehmende, massenhafte Verfügbarkeit digitalisierte Noten, etwa bei MuseScore ${ }^{40}$ oder bei UltimateGuitar, ${ }^{41}$ so scheint eine Übertragbarkeit der Methoden auf weitere musikalische Bereiche im Sinne eines Distant-Hearing-Ansatzes ${ }^{42}$ aus Perspektive der Digital Humanities mehr als plausibel.

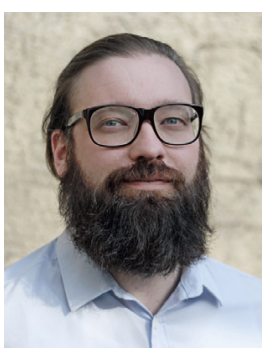

\author{
Manuel Burghardt \\ Juniorprofessur Computational Humanities \\ Institut für Informatik \\ Universität Leipzig \\ D-04009 Leipzig \\ burghardt@informatik.uni-leipzig.de
}

39 Jesser, Barbara: Interaktive Melodieanalyse: Methodik und Anwendung computergestützter Analyseverfahren in Musikethnologie und Volksliedforschung: typologische Untersuchung der Balladensammlung des DVA. Bern 1990.

40 MuseScore (notierte Musik): https://musescore.com/sheetmusic. 41 UltimateGuitar (Tabs): https://www.ultimate-guitar.com.

42 In Analogie zu Morettis (Anm. 3) Idee des Distant Reading, welche als alternativer Ansatz zum in der Literaturwissenschaft etablierten Close Reading verstanden werden kann. 